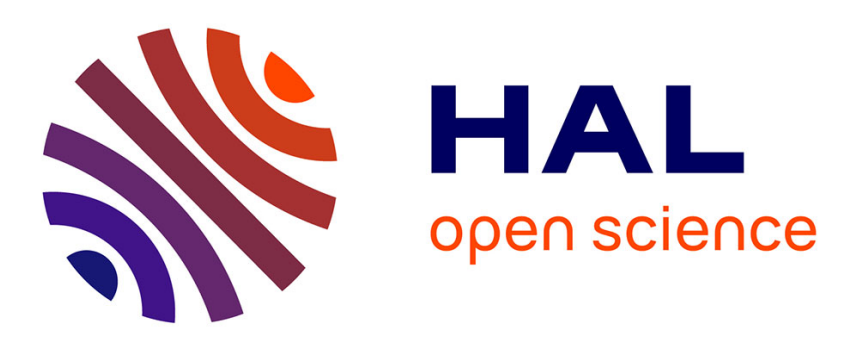

\title{
Numerical modelling of steady-state flow in 2D cracked anisotropic porous media by singular integral equations method
}

\author{
Ahmad Pouya, Minh-Ngoc Vu
}

\section{- To cite this version:}

Ahmad Pouya, Minh-Ngoc Vu. Numerical modelling of steady-state flow in 2D cracked anisotropic porous media by singular integral equations method. Transport in Porous Media, 2012, 93 (3), pp.475493. 10.1007/s11242-012-9968-1 . hal-00790536v2

\section{HAL Id: hal-00790536 \\ https://hal.science/hal-00790536v2}

Submitted on 20 Feb 2013

HAL is a multi-disciplinary open access archive for the deposit and dissemination of scientific research documents, whether they are published or not. The documents may come from teaching and research institutions in France or abroad, or from public or private research centers.
L'archive ouverte pluridisciplinaire HAL, est destinée au dépôt et à la diffusion de documents scientifiques de niveau recherche, publiés ou non, émanant des établissements d'enseignement et de recherche français ou étrangers, des laboratoires publics ou privés. 


\title{
Numerical modelling of steady-state flow in 2D cracked anisotropic porous media by singular integral equations method
}

\author{
Ahmad Pouya ${ }^{\mathrm{a}}$, Minh-Ngoc Vu $\mathbf{u}^{\mathrm{a}, \mathrm{b}, \mathrm{c}}$ \\ ${ }^{a}$ Université Paris-Est, Laboratoire Navier (UMR CNRS-IFSTTAR-ENPC), IFSTTAR, 58 bd Lefebvre, 75732 Paris, \\ France \\ ${ }^{b}$ BRGM/RNSC 45060 Orléans, Cedex 2 France \\ ${ }^{c}$ Le Quy Don Technical University, 100 Hoang Quoc Viet, Hanoi, Vietnam \\ ahmad.pouya@enpc.fr, vum@cermes.enpc.fr
}

\begin{abstract}
The equations governing plane steady-state flow in heterogeneous porous media containing curved-line intersecting cracks (Pouya and Ghabezloo 2010) and the potential solution obtained for these equations are considered here. The theoretical results are first completed for the mass balance at crack intersections points. Then, a numerical procedure based on a singular integral equations method is described concretely to derive this solution for cracked materials. Closed-form expressions of elementary integrals for special choice of collocation points lead to a very quick and easy numerical method. It is shown that this method can be applied efficiently to the study of the steady state flow in cracked materials with anisotropic matrix permeability and a dense distribution of curved-line intersecting cracks. Some applications of this method to the permeability of cracked materials are given.
\end{abstract}

Keywords: porous media, cracks, steady state flow, singular integral equations, effective permeability

\section{Introduction}

The discontinuities such as fractures and cracks have generally a great influence on the effective permeability of fractured rocks and porous materials. Some physical and engineering problems such as the contaminant transport in micro-cracked rocks or reservoir study in petroleum industry require the modelling of the fluid flow in porous media containing cracks or fractures. The problem of flow in a porous matrix containing cracks with mass exchange between matrix and cracks has been intensively investigated in recent years by different approaches (see the reviews given by Sánchez-Vila et al. (1995), Goméz-Hernández and Wen (1996) and Renard and de Marsily (1997)). Two-dimensional flow in and around a facture embedded by an infinite porous medium, under a constant pressure gradient, has in several cases derived analytically by means of some geometrical simplifications. In particular, the fracture has been sometimes modelled as a flattened ellipsoidal inclusion (Zimmerman, 1996) or as a two-dimensional lens (Mityushev and Adler, 2006) filled with a Darcy type material. In the 
present paper, we only consider the case of zero-thickness fractures with no pressure jump between the two faces of the fracture. This corresponds to an assumption of infinite transverse permeability of the fracture and excluding the cases where fractures act as an impermeable membrane like some cases of shear faults (Manzocchi et al. 2010). The effective permeability of materials with this kind of cracks has been studied by many numericalempirical approaches. A more rigorous approach based on the homogenization scheme has been given by Shafiro and Kachanov (2000), Dormieux and Kondo (2004) and Barthélémy (2009) in which the crack is assimilated to oblate ellipsoidal inclusions obeying to a Darcy's law. This powerful approach easily provides results for the effective permeability of 3D cracked materials, but it can not take into account crack intersections explicitly. Regarding the numerical modelling, the most difficult question is to generate a consistent mesh for a randomly fractured medium. A robust and automated algorithm was presented first by Koudina et al. 1998 to triangulate a polygonal fracture network; and then was extended for the fracture network and the embedding matrix as described by Bogdanov et al., 2003. The flow equations are then discretized by means of the finite volume method as to compute the effective permeability of fractured porous media (Bogdanov et al., 2003; Bogdanov et al., 2007; Mourzenko et al. 2011).

Following a different way, Liolios and Exadaktylos (2006) presented a mathematical formulation for plane steady-state flow in a cracked porous matrix where the cracks are considered as zero thickness discontinuity lines. However, their method, using complex number potentials, was restricted to isotropic matrix and excluded crack intersections. More recently, Pouya and Ghabezloo (2010) used a direct approach to model plane steady-state fluid flow in an anisotropic porous body containing curved line cracks with possible intersections. This approach provided a general potential solution for the fluid pressure in the matrix function of discharge along cracks. The flow in the cracks is modelled by a Poiseuille type law, a linear relation between the discharge and the pressure gradient along the crack. This model of cracks will be designated as Poiseuille crack to be distinguished from ellipsoidal cracks obeying to Darcy's law. Moreover, closed-form solutions were derived for the case of a single crack in an infinite matrix and applied to the problem of permeability upscaling.

In the present paper, we first complete the work of Pouya and Ghabezloo (2010) by the mass balance equation at crack intersection points. Then a numerical method is developed to solve the general system of singular equations for the case of an infinite body containing a dense family of curved line and intersecting cracks. This method is based on the resolution of the equations for a finite number of collocation points. A special choice of collocation points is given to simplify the computation. Besides, all the elementary integral terms are calculated explicitly. This leads to a highly efficient and fast calculation method. After presenting the method, a successful validation is first presented by comparing the numerical results obtained for a single superconductive crack with the closed-form solution for this case. Then, the flow is modelled around several curvilinear 
and intersecting cracks and the masse balance is checked carefully at intersection points. After that, the effective permeability of a material containing a random crack distribution, inspired from geological observations on a rock formation, is studied for the illustration of the method. Finally, the effective permeability of a periodic crack network is calculated with this method and the result is compared to those obtained by theoretical methods.

\section{Governing equations}

In this section, we briefly present the basic results obtained by Pouya and Ghabezloo (2010). An infinite heterogeneous porous body $\Omega$ containing a crack network is considered. The cracks are numbered by $m$ and denoted by $\Gamma^{m}$ (Fig. 1). The $\operatorname{crack} \Gamma^{m}$ is geometrically represented by a smooth curve $\underline{z}^{m}$ of the curvilinear abscise $s$. A crack can end in the matrix or intersect other cracks at its extremities. The intersection points of two or more cracks and the extremities of cracks ending in the matrix constitute a set of singular points denoted by $\mathrm{S}$. The set of points on the cracks are denoted by $\Gamma$, therefore, $\mathrm{S} \subset \Gamma$. The matrix corresponds to $\Omega-\Gamma$.

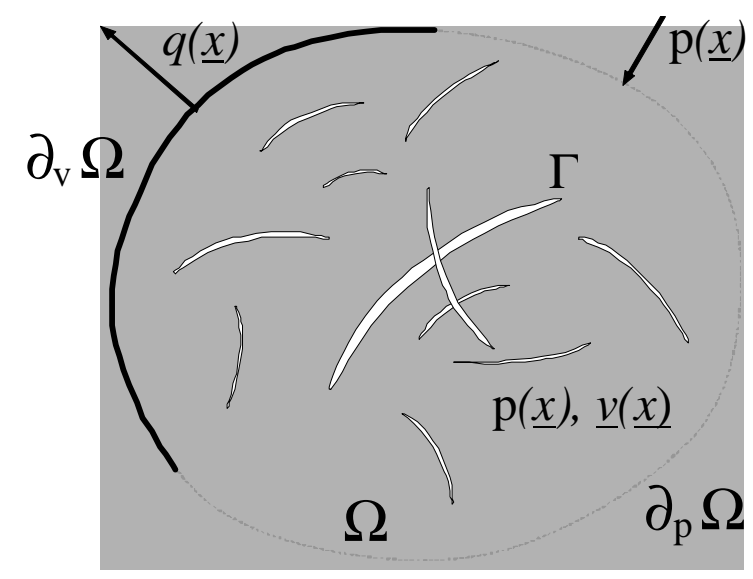

Fig. 1 Heterogeneous porous material containing micro-cracks

The fluid flow in the matrix is assumed to be governed by Darcy's law:

$$
\forall \underline{x} \in \Omega-\Gamma \quad \underline{v}(\underline{x})=-\mathbf{k}(\underline{x}) . \underline{\nabla} p(\underline{x})
$$

where, $\underline{v}(\underline{x})$ designates the fluid velocity, $\mathbf{k}$ the local permeability and $\underline{\nabla} p(\underline{x})$ the pressure gradient at $\underline{x}$.

In the absence of point sources, mass conservation in the matrix reads:

$$
\forall \underline{x} \in \Omega-\Gamma \quad \nabla \cdot \underline{v}(\underline{x})=0
$$

The flow model in the cracks is described commonly by a Poiseuille type law (Louis, 1969; Norton and Knapp, 1977; Witherspoon et al, 1980; Guéguen and Palciauskas, 
1994) in which the discharge $q$ in the crack is proportional to the pressure gradient along the crack line:

$$
\forall s \in \Gamma \quad q(s)=-c(s) . \partial_{s} p
$$

where $c$ is the hydraulic conductivity of the crack depending on its hydraulic aperture or its fill materials. According to the Poiseuille's law $c=e^{3} /(12 \mu)$ where $e$ is the hydraulic aperture of the crack and $\mu$ the fluid viscosity. Superconductive cracks correspond to the limite case of $(c \rightarrow \infty)$. In this case the pressure is constant along the crack curve.

The equation governing the crack-matrix mass exchange at regular points on the crack (excluding intersection points) is established by considering mass balance in a fragment of the crack comprised between abscises $s$ and $s+d s$ (Fig. 2a). It reads:

$$
\forall s \in \Gamma \quad \llbracket \underline{v}(\underline{z}) \rrbracket \cdot \underline{n}(s)+\partial_{s} q(s)=0
$$

In this equation, $\underline{z}$ is the point on the crack at the curvilinear abciss $s, \underline{n}(s)$ is the unit normal vector to the crack curve oriented from the side $\Gamma^{-}$to $\Gamma^{+}$(Fig. 2a) and $\llbracket \underline{v}(\underline{z}) \rrbracket=\underline{v}^{+}(\underline{z})-\underline{v}^{-}(\underline{z})$ is the discontinuity or jump of fluid velocity across the crack.

At crack intersections, analysing the mass balance in a circle centred at the intersection point, Pouya and Ghabezloo (2010) established the following relation for the fluid velocity at this point:

$$
\forall \underline{z} \in S \quad \underline{\nabla} \cdot \underline{v}(\underline{x})+\left(\sum_{j} q_{j}^{0}\right) \delta(\underline{x}-\underline{z})=0
$$

where, $q_{j}^{0}$ designates the outgoing discharge on the crack branch number $j, \underline{x}$ is a point in a small neighbourhood of $\underline{z}$ containing only $\underline{z}$ as singular point (Fig. 2b), and $\delta$ represents the Dirac distribution.

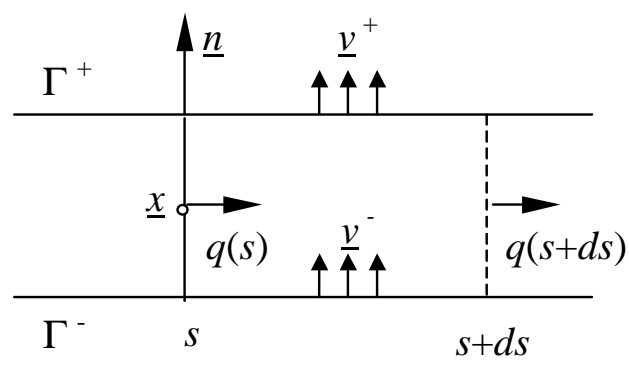

(a)

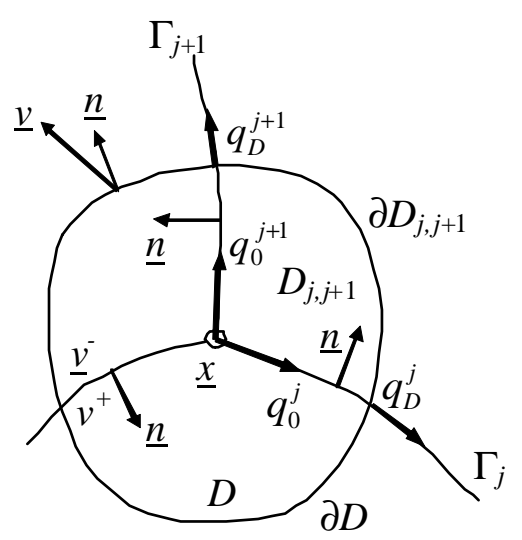

(b)

Fig. 2 a Crack-matrix mass exchange at a regular point on a crack. b Crack-matrix mass exchanges at an intersection point of several cracks 
The body is submitted at infinity to a pressure field $p_{\infty}(\underline{x})$ :

$$
\operatorname{Lim}_{\|\underline{x}\| \rightarrow \infty}\left[p(\underline{x})-p_{\infty}(\underline{x})\right]=0
$$

The field $p_{\infty}$ satisfies $\Delta p_{\infty}=0$. In the most general case of effective permeability modelling, $p_{\infty}$ corresponds to constant pressure gradient, i.e. $p_{\infty}(\underline{x})=\underline{A} . \underline{x}$ where $\underline{A}$ is a constant vector.

For the case of uniform matrix permeability $\mathbf{k}$, a general potential solution for these equations was rigorously established by Pouya and Ghabezloo (2010) with the following expression:

$$
p(\underline{x})=p_{\infty}(\underline{x})+\frac{1}{2 \pi \kappa} \sum_{\mathrm{m}} \int_{\Gamma_{\mathrm{m}}} q^{\mathrm{m}}(s) \frac{\underline{x}-\underline{z}^{\mathrm{m}}(s)}{\left\|\sqrt{\mathbf{k}^{-1}} \cdot\left[\underline{x}-\underline{z}^{\mathrm{m}}(s)\right]\right\|^{2}} \cdot \mathbf{k}^{-1} \cdot \underline{t}^{\mathrm{m}}(s) d s
$$

In this relation, $\underline{x}$ is the current point in the porous body, $\underline{z}^{\mathrm{m}}(s)$ is the point on the crack $\Gamma^{\mathrm{m}}$ at the curvilinear abscise $s, q^{\mathrm{m}}(s)$ is the discharge along the crack $m, \underline{t}^{\mathrm{m}}(s)$ is unit vector tangent to this crack at the point $\underline{z}^{\mathrm{m}}(s)$ and $\kappa$ is the square root of determinant of $\mathbf{k}: \quad \kappa=\sqrt{|\mathbf{k}|}$. If the discharge $q(s)$ in (7) is replaced by its expression function of $p(s)$, for instance by (3), and the collocation point $\underline{x}$ is taken on the cracks, then a system of equations is obtained that allows us to determine the pressure $p$ on the crack network. It is worth noting that this system of equation involves only the pressure on the crack lines and thus reduces the dimension of the problem from 2 to 1 . This simplifies considerably the numerical resolution method in the same way that Boundary Elements method simplifies the numerical resolution compared to Finite Elements or Finite Volume methods.

\section{Mass balance at intersection points}

A deeper mathematical analysis of the mass balance condition at intersection points allows going further than equation (5) and demonstrates that at these points we have:

$$
\sum_{j} q_{j}^{0}=0
$$

This relationship has an important physical interpretation and consequences for numerical modelling that will be detailed further. To demonstrate it, let us consider a small domain $D$ surrounding an intersection point $\underline{z}$ with its boundary designated by $\partial D$ (Fig. 2b). If there are $J$ intersecting cracks at $\underline{z}$, then $D$ is divided into $J$ sub-domains $D_{j, j+1}$ bounded by the cracks $\Gamma_{j}$ and $\Gamma_{j+1}$ (with the convention $\Gamma_{J+1}=\Gamma_{1}$ to have cyclic notation). The mass exchange between $D$ and the matrix contains two parts: one by flow through the matrix on $\partial D$ with fluid velocity $\underline{v}$, and the other by the cracks $\Gamma_{j}$ at their intersection with $\partial D$ where discharge is designated by $q_{j}^{D}$. Therefore, the mass balance for this domain reads:

$$
\int_{\partial D} \underline{v} \cdot \underline{n} d s+\sum_{j} q_{j}^{D}=0
$$


Now, we consider the mass balance in the subdomain $\partial D_{j, j+1}$. It reads:

$$
\int_{\partial D_{j, j+1}} \underline{v} \cdot \underline{n} d s-\int_{\Gamma_{j}^{D}} \underline{v}^{+} \cdot \underline{n} d s+\int_{\Gamma_{j+1}^{D}} \underline{v}^{-} \cdot \underline{n} d s=0
$$

where, $\Gamma_{j}^{D}$ represents the portion of $\Gamma_{j}$ included in $D, \underline{n}$ is the unit normal vector on the integration line. On $\partial D_{j, j+1}, \underline{n}$ represents the outward unit normal, whereas on $\Gamma_{j}$, it is oriented from the surface $\Gamma_{j}$ to the domain $D_{j, j+1}$. On $\Gamma_{j}$ the fluid velocity is discontinuous: $\underline{v}^{-}$and $\underline{v}^{+}$represent the velocity at respectively the negative and positive sides of this surface orientated by $\underline{n}$ (Fig. 2b). This convention determines the sign of the two last integrals in (10). When summing this equation on $j$, the contribution of the two last integrals can be transformed into expressions involving the jump of $\underline{v}$ on $\Gamma_{j}$. Hence, the following equation is then found:

$$
\int_{\partial D} \underline{v} \cdot \underline{n} d s-\sum_{j} \int_{\Gamma_{j}^{D}} \llbracket \underline{v} \rrbracket . \underline{n} d s=0
$$

Now, by using (4) the second integral in this equation can be changed to write:

$$
\sum_{j} \int_{\Gamma_{j}^{D}} \llbracket \underline{v} \rrbracket \cdot \underline{n} d s=-\sum_{j}\left(q_{j}^{D}-q_{j}^{0}\right)
$$

Introducing (12) and (11) into (9) results in (8).

The physical interpretation of (8) is that the masse balance condition at crack intersection points is independent from the crack-matrix mass exchange. This relation is the same that one would find for a crack network in impermeable material. Moreover, this result also allows extending the Cauchy integration to intersection points by elimination of the singular contribution of different crack branches at these points to the integral (7). This equation will be verified numerically in Section 5.

\section{Numerical resolution}

By replacing $q(s)$ in (7) by its expression (3), a system of integro-differential equations is obtained and then solved in order to determine the pore pressure and the fluid flow fields. This system allows us to investigate in a first stage only the pressure along crack lines and determining it by resolving the system of singular integral equations. In the second stage, the integral equation (7) with the known expression of the discharge $q(s)$ will be used to calculate the pressure field at an arbitrary point of the matrix. It is worth noting that the values of $q(s)$ in the first stage can be directly used for permeability upscaling and the second stage is not necessary for this purpose. This is an interesting feature of the numerical method presented here as it will be seen in section 6 .

The principle for resolving the singular integral equations by collocation method can be found in Bonnet (1995). We propose in this paper a special choice of interpolation functions and collocation points as well as analytical expressions for elementary 
integrals that lead to an efficient and fast resolution method. The numerical method presented in the following assumes a constant value for the cracks conductivity $c$ but it can be easily extended to variable conductivities for cracks.

\subsection{Discretization and Interpolation}

As a consequence for the theoretical formulae (7), numerical calculation only requires a mesh generation on the cracks which must be consistent by taking into account crack intersection points. Thus, cracks are discretized by a number of intervals, called in the sequel «elements». The set of intervals on all cracks define globally $N$ elements numbered by $n$ and denoted by $E_{n}(1 \leq n \leq N)$. With these notations, Eq. (7) can be written as:

$$
p(\underline{x})=\underline{A} \cdot \underline{x}+\sum_{n=1}^{N} I_{n}
$$

where,

$$
I_{n}(\underline{x})=\int_{E_{\mathrm{n}}} q^{\mathrm{n}}(s) \frac{\underline{x}-\underline{z}^{\mathrm{n}}(s)}{\left\|\sqrt{\mathbf{k}^{-1}} \cdot\left[\underline{x}-\underline{z}^{\mathrm{n}}(s)\right]\right\|^{2}} \cdot \mathbf{k}^{-1} \cdot \underline{t}^{\mathrm{n}} d s
$$

In order to define the interpolation functions of variable on the elements, we distinguish two types of elements: current element on the crack and extremity elements where the cracks end in the matrix. On the current elements, a linear interpolation of pressure is used; hence, $q$ is constant on the element (Fig. 3). For extremity elements, general theoretical results concerning the velocity field singularity close to a crack tip leads to suppose that $\underline{v}$ varies as $r^{1 / 2}$ around the crack extremity points (Muskhelishvili, 1953). Besides, based on the analytical solutions of fluid flow in superconductive cracks (Pouya and Ghabezloo, 2010), we choose an interpolation function for discharge $q(s)$ with a variation as $s^{1 / 2}$ where $s$ is the distance from crack extremity. This leads to a variation as $s^{3 / 2}$ for $p(s)$ on the extremity elements. We designate by $L$ the length of the element and by $p_{1}$ and $p_{2}$ its nodal pressures at curvilinear abscises $s=0$ and $s=L$. Then the interpolation functions for the pressure and discharge on current elements, left extremity elements (tip at $s=0$ ) and right extremity elements (tip at $s=L$ ) take, respectively, the following expressions:

$$
\begin{aligned}
& p(s)=p_{1}+\frac{p_{2}-p_{1}}{L} s ; \quad q(s)=-c \frac{p_{2}-p_{1}}{L} \\
& p(s)=p_{1}+\frac{p_{2}-p_{1}}{\sqrt{L}^{3}} \sqrt{s}^{3} ; \quad q(s)=-a \sqrt{s}
\end{aligned}
$$




$$
p(s)=p_{2}-\frac{p_{2}-p_{1}}{\sqrt{L}^{3}} \sqrt{L-s}^{3} ; q(s)=-a \sqrt{L-s}
$$

where:

$$
a=\frac{3 c}{2} \frac{p_{2}-p_{1}}{\sqrt{L}^{3}}
$$

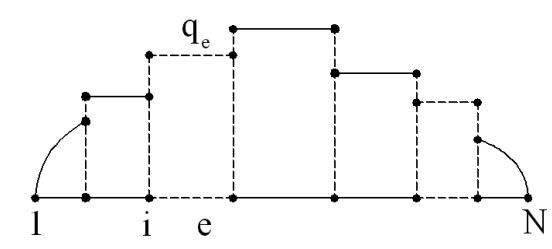

(a)

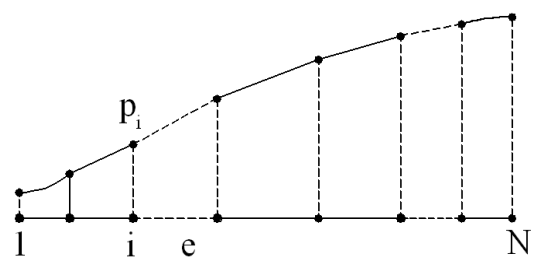

(b)

Fig. 3 Interpolation of the discharge (a) and pressure (b) a long a discretized crack

\subsection{Choice of collocation points}

The collocation method consists in enforcing exactly the singular integral equation (7) at a finite number of points called collocation points. In its simplest form and in most studies in the literature, these collocation points are chosen to be the nodes used to discretize the geometry. Herein, we propose another way to choose the collocation points that easily allows the introdution of analytical expressions for the elementary integrals and thus the simplification of the calculation. At first, a collocation point is taken per element and if the collocation point $\underline{x}$ is on the element $E_{n}, I_{\mathrm{n}}(\underline{x})$ becomes a singular integral. For the current element, a collocation point $\underline{x}_{n}$ is placed naturally in the middle of the element because this implies:

$$
I_{n}\left(\underline{x}_{n}\right)=0
$$

This equation results from the linearity of pressure interpolation on the current elements and simplifies the calculations. It suggests also choosing the collocation point on the extremity element such that the same equation (19) will be obtained.

Consider a left-extremity element $E_{n}$ with endpoints $\underline{z}_{1}$ and $\underline{z}_{2}$ and take the collocation point:

$$
\underline{x}=\underline{z}\left(s_{x}\right)=\underline{z}_{1}+\left(s_{x} / L\right)\left(\underline{z}_{2}-\underline{z}_{1}\right)
$$


with $0<s_{\mathrm{x}}<L$. Substituting Eqs.(16), (18) and (20) into Eq.(14), then $I_{\mathrm{n}}(\underline{x})$ can be integrated explicitly. As a result, equation (19) is fulfilled for a value of $s_{x} / L=\xi$ where $\xi$ is the solution of the equation:

$$
\log \frac{1+\sqrt{\xi}}{1-\sqrt{\xi}}=\frac{2}{\sqrt{\xi}}
$$

Numerical computation leads to $\xi \approx 0.694817$. In a similar way, the condition $s_{x} / L=1-\xi$ is obtained with the same numerical value for $\xi$ for right-extremity elements.

In conclusion, on the element $E_{\mathrm{n}}$, a collocation point $\underline{x}_{\mathrm{n}}=\underline{z}_{1}+\xi_{n}\left(\underline{z}_{2}-\underline{z}_{1}\right)$ is chosen with, $\xi_{\mathrm{n}}=\xi, \xi_{\mathrm{n}}=0.5$ and $\xi_{n}=1-\xi$ for respectively left-extremity, current and right-extremity elements.

It should be noted that depending on the crack network connectivity, the number of elements can be smaller than the number of nodes. Hence, it is not generally sufficient to take one collocation point per element to solve the system of equations that has the nodal pressures as unknown variable because the number of equations can be smaller than the number of unknowns. The difference between the number of nodes and elements depends on the connectivity properties of the crack network and is not easy to determine. Nevertheless, a summary count of nodes and elements could help to determine an optimum choice of collocation points. As a matter of fact, each element is always related to two nodes, whereas, except for crack extremity points, a node is related to two or more elements. Thus, the number of elements is more than the number of nodes that are not crack extremities. Therefore, if, to the set collocations points $\underline{x}_{\mathrm{n}}$ defined here-above (the same number than the elements), we add the crack extremity points, the number of collocation points will become always greater than the number of nodes. In this way, we can have more equations than unknowns, and then the least square fitting method will be required to solve the problem. This method will be presented later in the paper.

\subsection{Explicit expression for elementary integrals}

In the literature on boundary elements method, computation of regular elementary integrals is usually based, as well as in finite element method, on the use of Gaussian quadrature formulas. Contrariwise, the Cauchy Principal Value definition for the singular elementary integral is the standard technique in the boundary integral analysis (Guiggiani, 1991). However, there are still other techniques available to deal with the singular integral such as: regularization technique (Tanaka et al. 2002) that permits treating weak singularity; boundary limit approach (Sutradhar et al. 2008) that defines the boundary equation as a limit case of $\underline{x}$ approaches the boundary from either interior or exterior and which is efficient for symmetric Galerkin boundary elements method. Otherwise, the numerical difficulty appears when $\underline{x}$ is too close to $E_{n}$. In this case, the integrals are not strictly singular but nearly singular and can be computed by some transformation techniques that allow the concentration of Gauss's points in the vicinity of the orthogonal projection of $\underline{x}$ on the considered element. In this study, all elementary integrals are explicitly computed by an analytical procedure which aims to a high speed 
convergence of numerical computation and avoids also the numerical integration difficulties.

According to the relative position of the point $\underline{x}_{\mathrm{c}}$ and the element $E_{n}$, we can distinguish five types of elementary integrals $I_{\mathrm{n}}\left(\underline{x}_{\mathrm{c}}\right)$ to be calculated:

(i) $\underline{x}_{\mathrm{c}}=\underline{x}_{\mathrm{n}}$ is the collocation point defined here above on the interval $E_{\mathrm{n}}$,

(ii) $\underline{x}_{\mathrm{c}}$ is the extremity point on the extremity element $E_{\mathrm{n}}$,

(iii) $\underline{x}_{\mathrm{c}}$ is an intersection point

(iv) $\underline{x}_{\mathrm{c}}$ is on the element $E_{\mathrm{n}}$ but is not an extremity nor endpoint,

(v) $\underline{x}_{\mathrm{c}}$ is not on $E_{\mathrm{n}}$.

For the first case, we have already chosen $\underline{x}_{\mathrm{n}}$ verified $I_{\mathrm{n}}\left(\underline{x}_{\mathrm{n}}\right)=0$. For the second case, the integrant function is singular at the point $x_{\mathrm{c}}$, but remains integrable because its singularity is the order of $s^{-1 / 2}$. In the third case, $\underline{x}_{\mathrm{n}}$ is an intersection point between several cracks or common points between several elements $E_{\mathrm{n}}$. Each $I_{\mathrm{n}}\left(\underline{x}_{\mathrm{c}}\right)$ is singular in this case, nevertheless, the sum of singularities vanishes because of the mass balance (8). For the fourth case, the integrant function is singular at $\underline{x}_{c}$, but can be integrated in Cauchy principal value sense. Finally, the integrand function is not singular in the last case. For determination of the nodal pressure, or for the first stage of our numerical method (see the beginning of Section 4), the system of equations derived from (13) involves only the first and second cases listed above. In these cases, $I_{\mathrm{n}}\left(\underline{x}_{\mathrm{c}}\right)$ can be expressed by the analytical expressions. Hence, equation (13) leads to a matrix equation in which all components of the matrix are computed analytically. This provides a very fast calculation method for determining the nodal pressures in the first stage. In the case (iii) listed above, $x_{\mathrm{c}}$ is a nodal point and its pressure in deduced from the results of the first stage. The pressure for the case (iv) is deduced by interpolation functions (15) to (17) from the nodal pressure. For the last case $(v)$, analytical expressions for elementary equations can also be derived that simplify the calculations. Analytical expressions of different cases of elementary integrals are given in Appendix.

\subsection{Construction and resolution of linear system of equations}

The set of $M$ scalar equations (13) for $N$ nodal pressure unknowns have the following matrix structure:

$$
\mathrm{F}=\mathbf{H P}=\mathrm{C}+\mathbf{G P}
$$

where, $\mathrm{F}(M \times 1)$ is the column of pressure values at collocation points, $\mathrm{P}(N \times 1)$ is the column of unknown nodal pressure, $\mathrm{C}(M \times 1)$ is column of infinite pressure field $p_{\infty}$ applied to collocation points, $\mathbf{H}(M \times N)$ is established from the interpolation functions in Eqs.(15)-(17), $\mathbf{G}(M \times N)$ is computed from the elementary integrals in right-hand side of equation (7) which is set up through the assembly operation that is carried out by two nested loops: the first one is over all collocation points and the second one over all elements.

As a consequence, the system (22) consists of $M$ equations and $N$ unknowns with $M>N$. However, an approximate solution can be computed by using the least squares fitting 
method which is performed by minimizing $|(\mathbf{H}-\mathbf{G}) \mathrm{P}-\mathrm{C}|^{2}$ on the $\mathrm{P}$ values. This leads to the equation:

$$
\mathrm{KP}=\mathrm{Y}
$$

where:

$$
\mathbf{K}=(\mathbf{H}-\mathbf{G})^{\mathrm{T}}(\mathbf{H}-\mathbf{G}) ; \mathbf{Y}=(\mathbf{H}-\mathbf{G})^{\mathrm{T}} \mathbf{C}
$$

In this way, finally, the system of equations (13) is reduced to a linear system of $N$ equations on $N$ unknown nodal pressures.

\section{Numerical examples}

In this section, we present some examples that illustrate the numerical method obtained in precedent sections. The algorithm described in precedent section was been implemented in a Fortran code that generates automatically a consistent mesh to discretize the crack network. Then, as described here above, the nodal pressures are determined from (24) in a first stage and inserted in (13) to determine the pressure at an arbitrary point.

\subsection{Straight single crack in an infinite plane}

In order to validate the numerical solution, the fluid flow around a single straight crack in an infinite homogeneous matrix was modeled and compared to the analytical and semi-analytical results obtained by Pouya and Ghabezloo (2010). Consider an infinite matrix, with uniform and isotropic permeability $k$, containing a straight crack of length $2 L$ and conductivity $c$, occupying the interval $[-L, L]$ on the $x$-axis of coordinate system. This infinite body is submitted to a farfield pressure with a uniform gradient $\underline{A}=(a, 0)$ parallel to the crack:

$$
p_{\infty}(\underline{x})=a \cdot x
$$

The following dimensionless parameters are introduced:

$$
\lambda=\frac{c}{2 \pi k L}, \quad X=\frac{x}{L}, \quad P_{\lambda}(X)=\frac{p(x, 0)}{a L}
$$

Fig. 4 displays, for different values of $\lambda$, the pressure and the discharge obtained along the crack line. Since the problem is symmetric with respect to the $y$-axis, only the solution on the right half-crack $(0 \leq X \leq 1)$ is presented in the figure. It is important to note that, for every value of $\lambda>0$, at the crack tip, the discharge $q$ as well as the pressure gradient in the crack are null. The pressure field obtained for the limit case of superconductive crack $\left(c \rightarrow \infty\right.$ or $\lambda \rightarrow \infty$, numerically $\left.\lambda=10^{5}\right)$ is presented in Fig. 5 . This figure shows the singularity of the pressure gradient in the neighbourhood of the crack tip. The numerical results obtained here are in perfect agreement with the semi-analytical or close-formed solutions given by Pouya and Ghabezloo (2010) (Fig. 4 - right). 

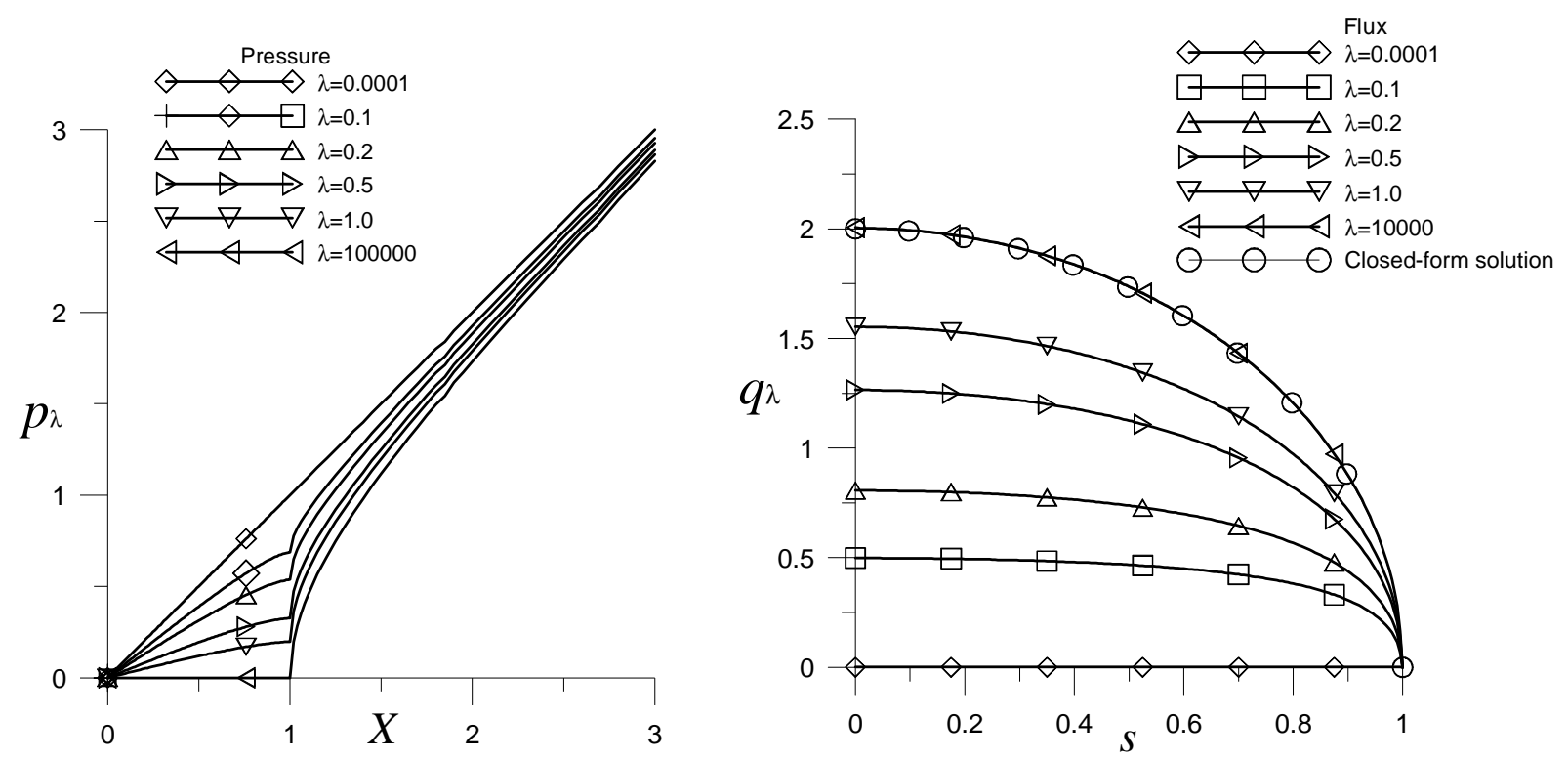

Fig. 4 Pressure and discharge along the crack line plotted for different values of $\lambda$. Left: pressure $P_{\lambda}$ along the $x$-axis, right: discharge $q$ in the crack $(0<X<1)$

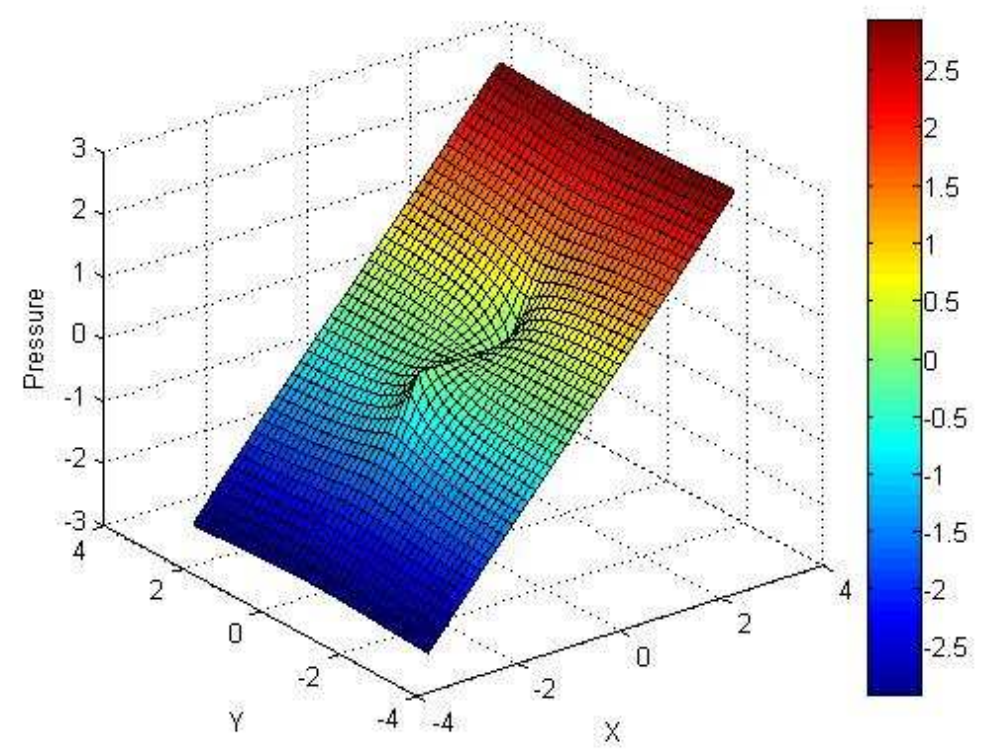

Fig. 5 Pressure field $p(x, y)$ in the plane of the crack for a uniform pressure gradient at infinity parallel to the crack ( $X$-axis in the figure).

\subsection{Curvilinear cracks with intersection}

The following example of several straight or curvilinear intersecting cracks allows us to illustrate the benefit of the new numerical method compared to previous works, and also to numerically check the mass balance equation (8). The geometry of the cracks is presented in the Fig. 6, with details for elements surrounding the intersection point on 
the right. The pressure field has been calculated here for an anisotropic matrix $\left(k_{x x}=2\right.$, $\left.k_{y y}=0.5\right)$ under the limit condition (25). The pressure field obtained in the matrix is presented in the left of Fig. 6. This example illustrates two improvements of the method compared to that presented by Liolios and Exadaktylos (2006): extension to intersecting cracks and to anisotropic matrix.

Note that the limit condition of pressure is given by $p(x)=a x$ at infinity and not on the boundary of the domain presented in the figure. On this boundary the pressure is affected by the presence of the cracks. This shows another advantage of the method: in numerical calculations for determination of the effective permeability of cracked materials, one often seeks for the flow in a family of cracks in an infinite matrix subjected at infinity to a uniform pressure gradient. This is the concept of an inclusion in an infinite matrix. But to determine numerically the flow field, generally an REV (Representive Element Volume) is considered and a pressure $p(x)=a x$ is imposed on the boundary of this REV (Long et al., 1982, Pouya and Fouché, 2009). This does not correspond to the theoretical condition of a farfield pressure gradient. The method presented here, based on equation (7), allows naturally imposing a uniform pressure gradient at infinite boundary.

Here is also an occasion to check if the numerical results satisfy well the mass balance (8) at the intersection points. Note that equation (8) has not been included in the system of equations determining the numerical results and then mass balance (8) is not an $a$ priori condition imposed on these results.

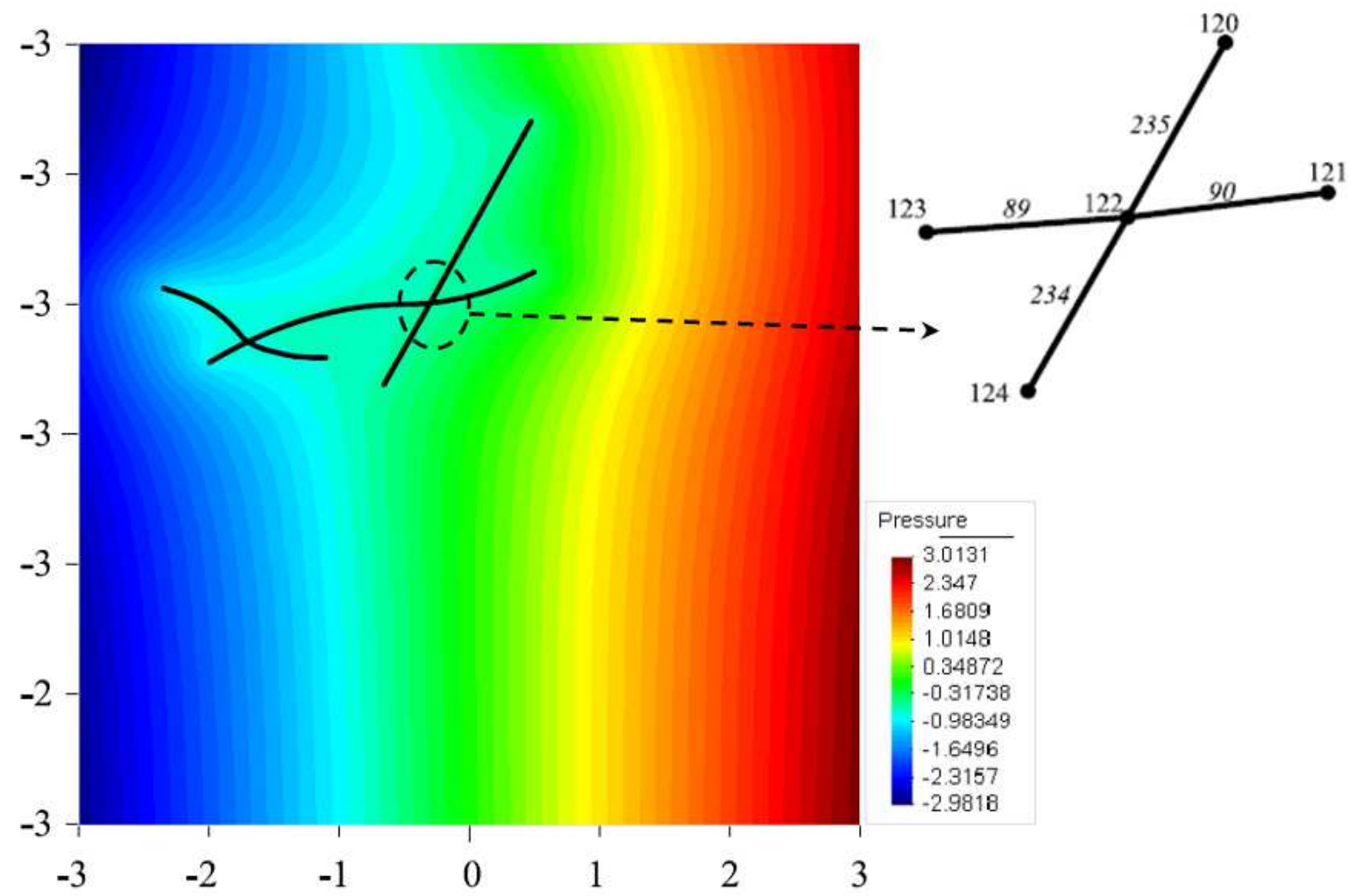

Fig. 6 The Pressure field $p(x, y)$ in the anisotropic matrix 
However, the numerical discharges obtained on the elements related to the intersection point in the right-side of Fig. 6 (the node $j=122$ ), show that this mass balance is very accurately satisfied. As a matter of fact, let the error estimation be defined by:

$$
E=\frac{\left|\sum_{b} q_{j}^{b}\right|}{\left|q_{j}^{b}\right|}
$$

where $q_{j}^{b}$ is the discharge outgoing from the node $j$ on the branch $b$ (one of the four elements in the case considered here), and $\widehat{\mid q_{j}^{b}} \mid$ is the average absolute value of theses discharges. In the case considered here above, we found at the intersection point $j=122$ $E \approx 0.004$ and $E \approx 0.012$ for an isotropic and anisotropic matrix, respectively Thus, the mass balance is fulfilled with a good accuracy. The same result is obtained for all the intersection points that were analyzed.

\section{Application to the permeability of cracked porous media}

The numerical method established here above specially becomes interesting and efficient compared to other methods in cases where a great number of cracks are present. We show in this section its application to compute the permeability of cracked materials which needs the modelling of the flow in the presence of a crack distribution.

A homogeneous domain $\Omega$, containing a population of internal curvilinear cracks $\Gamma_{m}$, is under the influence of the linear pressure condition $p(\underline{x})=\underline{A} . \underline{x}$ applied on its boundary $\partial \Omega$. A general and classical result for the case of cracks with zero or negligible thickness is that the effective permeability $\mathbf{k}^{\mathrm{e}}$ is the sum of the matrix permeability $\mathbf{k}$ and a contribution of cracks $\mathbf{k}^{\mathrm{c}}$;

$$
\mathbf{k}^{\mathrm{e}}=\mathbf{k}+\mathbf{k}^{\mathrm{c}}
$$

The crack contribution $\mathbf{k}^{\mathrm{c}}$ can be deduced from the balance of discharge in the cracks under a farfield pressure gradient applied to the matrix from the following relation (Bogdanov et al., 2003; Liolios and Exadaktylos, 2006; Pouya and Ghabezloo, 2010):

$$
\frac{1}{\Omega} \sum_{m} \int_{\Gamma_{m}} q \underline{t} d s=-\mathbf{k}^{c} \cdot \underline{A}
$$

In this formula, $\underline{A}$ is the pressure gradient imposed on the boundary $\partial \Omega$ that contains the cracks $\Gamma_{\mathrm{m}}$. It is sufficient to compute the left-side of (29) for two different directions of $\underline{A}$ to be able to determine $\mathbf{k}^{\mathrm{c}}$. The result can be compared to that predicted by approximate closed-form solutions for the case of straight cracks. Let $\Gamma^{m}$ designate a family of cracks with length $2 L^{m}$, orientation $\underline{t}^{m}$, conductivity $c^{m}$ and density (number of crack centres per unit area) $\rho^{m}$. In the case of randomly oriented cracks, $\mathbf{k}^{\mathrm{c}}$ is isotropic:

$$
\mathbf{k}^{\mathrm{c}}=\mathrm{k}^{\mathrm{c}} \boldsymbol{\delta}
$$


and then $\mathbf{k}^{\mathrm{e}}=k^{\mathrm{e}} \boldsymbol{\delta}$ where, according to Pouya and Ghabezloo (2010), the Mori-Tanaka and self consistent estimates, $k^{\mathrm{e}}$ are respectively given by (31a) and (31b):

$$
\begin{aligned}
& k^{e}=k+\sum_{m} L^{m} \rho^{m} \frac{\pi \kappa L^{m} c^{m}}{2 c^{m}+\pi \kappa L^{m}} \\
& k^{e}=k+\sum_{m} L^{m} \rho^{m} \frac{\pi \kappa^{e} L^{m} c^{m}}{2 c^{m}+\pi \kappa^{e} L^{m}}
\end{aligned}
$$

with $\kappa=\sqrt{|\mathbf{k}|}, \kappa^{e}=\sqrt{\left|\mathbf{k}^{e}\right|}$. In this case $\kappa=k$ and $\kappa^{e}=k^{e}$.

For a network of parallel cracks, with unit vector $\underline{t}$, we have:

$$
\mathbf{k}^{\mathrm{c}}=\mathrm{k}^{\mathrm{c}} \underline{t} \otimes \underline{t}
$$

and then:

$$
\mathbf{k}^{\mathrm{e}}=k \underline{n} \otimes \underline{n}+k^{\mathrm{e}} \underline{t} \otimes \underline{t}
$$

where, the Mori-Tanaka and self consistent estimates of $k^{\mathrm{e}}$ are deduced respectively from (34a) and (34b):

$$
\begin{aligned}
& k^{e}=k+2 \rho L \frac{\pi c L \kappa}{2 c+\pi L \kappa} \\
& k^{e}=k+2 \rho L \frac{\pi c L \kappa^{e}}{2 c+\pi L \kappa^{e}}
\end{aligned}
$$

where, $\kappa^{e}=\sqrt{k \cdot k^{e}}$. Thus, the self-consistent scheme (34b) leads to the third-degree equation for the variable $k^{\mathrm{e}}$.

The dilute Mori-Tanaka estimations (31a) and (34a) do not take into consideration the interaction between cracks whereas this interaction is implicitly taken into account in some approximate forms of the self consistent scheme and fully in the numerical modelling. In the following, the theoretical results will be compared to the results obtained by the numerical method in the two cases that of porous media containing a distribution of randomly oriented cracks and that of a regular network of parallel cracks.

\subsection{Randomly oriented cracks}

A geological rock formation is considered containing several families of fractures. Each family of fractures is characterized by an average length, a mean orientation and a density that are determined from in situ geological observations (Billaux, 1990; Gervais, 1993). The rock formation is represented by an infinite porous matrix with uniform and isotropic permeability and containing several families of cracks randomly generated by stochastic laws. A domain of $10 \times 10$ (Fig. 7 - left) in the cracked matrix is used for the numerical modelling. In this domain, fractures are generated according to statistical laws 
reproducing the geological data. This domain is submitted to the boundary conditions (25). A smaller area is taken in which the pressure field is computed and used for the equivalent permeability calculation. (Fig. 7 - right). The numerical method is very easy to use since it does not require meshing the whole surface as it would be required for Finite Element or Finite Volume methods. The pressure field obtained by this method is represented in Fig. 7 - right. The conductivity of cracks relative to the matrix permeability is given by $\lambda=1$ where dimensionless variable $\lambda$ is defined by (26). For a crack family of density (number of crack centres per unit area) $\rho=2.5$, average length $L=$ 0.35 and of uniformly distributed orientation (so globally isotropic), the Mori-Tanaka estimate (31a) provides $k^{\mathrm{e}} / k=1.38$, the self consistent estimate, $k^{\mathrm{e}} / k=1.53$ and the numerical calculation $k^{\mathrm{e}} / k=1.59$. The difference between the numerical and MoriTanaka estimation reveals the effect of the interaction between cracks. The selfconsistent estimate takes approximately the crack interaction into account and is, for this reason, closer to the numerical result.

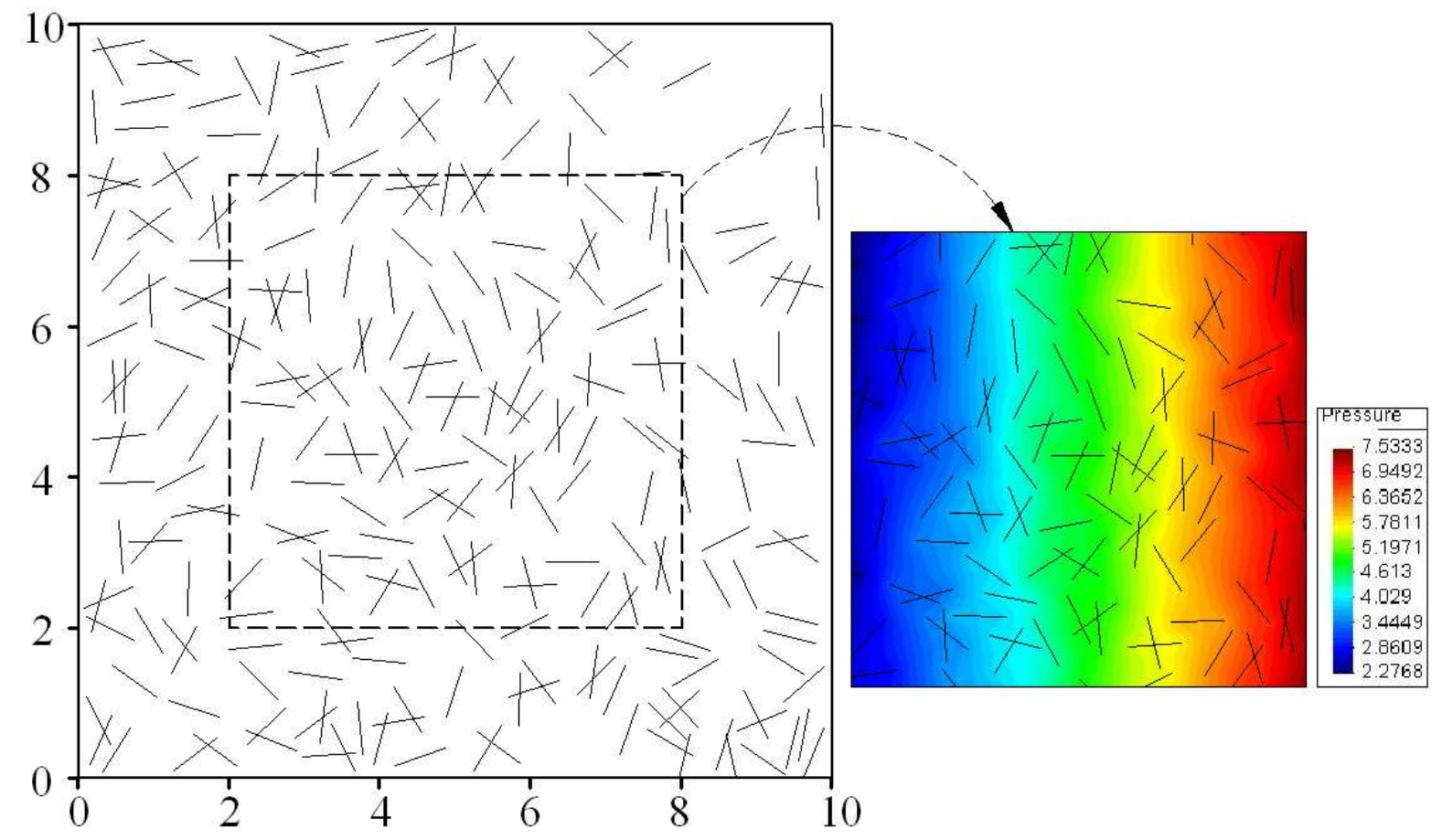

Fig. 7 Cracks network in geological rock formation

\subsection{Periodic distribution cracks}

An infinite porous matrix containing a periodic distribution of cracks parallel to the $x$ axis is considered (Fig. 8). The matrix has a uniform and isotropic permeability $k$, and is submitted to the farfield pressure gradient (25). The cracks have the length $2 L$ and the distance $2 b$ between their extremities in the $x$-direction and $h$ between their centres in the $y$-direction. The crack density is then: 


$$
\rho=\frac{N}{\Omega}=\frac{1}{2 h(b+L)}
$$

where, $N$ is number of cracks on $\Omega$. The mean value of $\int_{\Gamma} q d s$ is numerically computed on an array of $(m \times m)$ cracks at the centre of the domain with $m=1,2,3 \ldots$ Greater is $m$, more accurately the interaction between cracks is taken into account. As predicted by the formula (33), the effective permeability is changed only in the direction parallel to cracks.

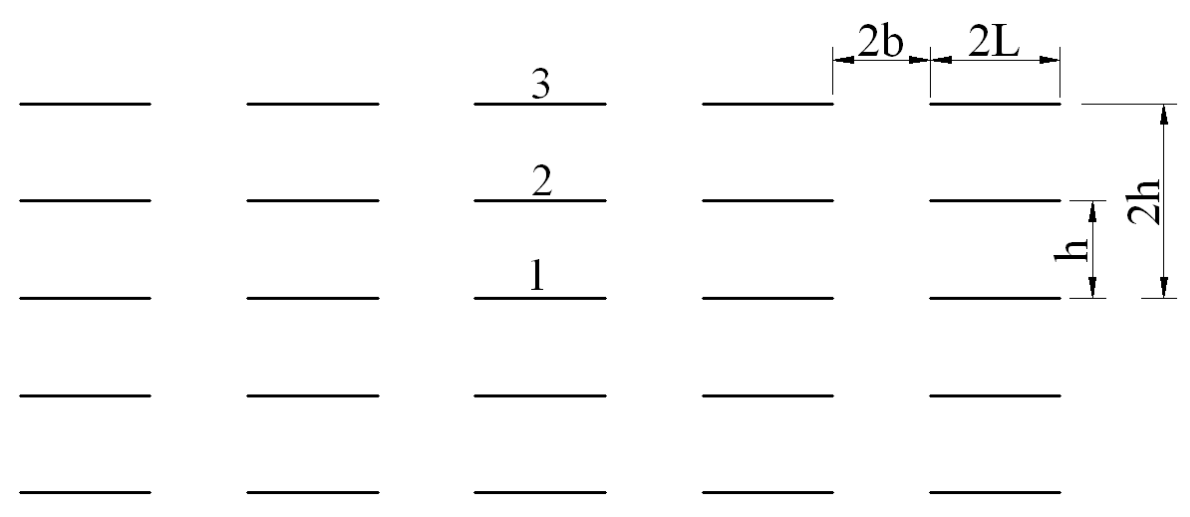

Fig. 8 Periodic distribution of parallel cracks in infinite porous media

Fig. 9 depicts the variation of $k^{\mathrm{e}}$ as a function of the crack density $\rho$ for different values of $\lambda$. The equivalent permeability $k^{\mathrm{e}}$ obviously increases with the density $\rho$ and with the crack conductivity represented by $\lambda$. The evolution of $k^{\mathrm{e}}$ with $\rho$ is not linear due to the cracks interactions. It is interesting to compare these results to theoretical estimates.

Erreur ! Source du renvoi introuvable. Effective permeability of direction x plotted as a function of crack density $\rho$ for different dimensionless variable $\lambda$

Fig. 10 displays the effective permeability computed by the numerical method compared to Mori-Tanaka and self consistent estimates. The three curves begin with the same slope at $\rho=0$, and this shows that the theoretical estimates give the exact variation of the permeability at first order development for small $\rho$ values. The dilute Mori-Tanaka estimate remains a linear function of $\rho$ because it does not take into consideration the cracks interaction. It can be noticed that the self-consistent estimate, that is supposed to take into account in some extent the cracks interactions, is strangely less accurate that the Mori-Tanaka estimate. This result is in contrast with that obtained in precedent section for randomly oriented cracks. However, it is well known that the self-consistent scheme improves the estimation only in the case of a random orientation of the heterogeneity (Dormieux and Kondo, 2004). It can be shown that the in the case of parallel cracks must lead to a smaller equivalent permeability than that one obtained by the Mori-Tanaka estimate, and to prove that the numerical results presented here give the 
good trend of equivalent permeability versus density. This can be done by using the solution obtained for the pressure field around a single crack as shown in Fig. 10.

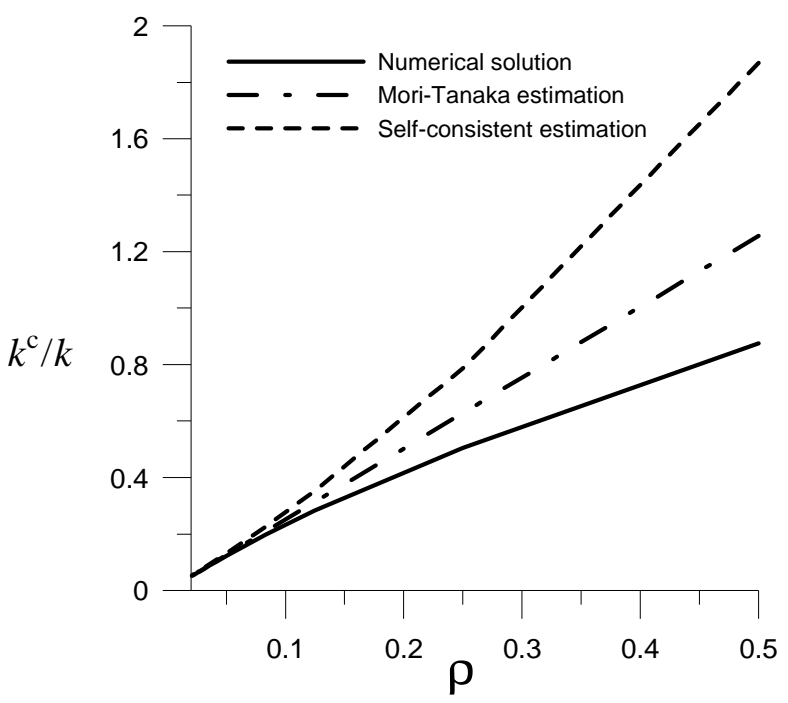

Fig. 9 Comparison of effective permeability in the direction $x$ obtained by three different ways: numerical method and Mori-Tanaka and self-consistent estimations $(\lambda=1)$

Fig. 10 displays the pressure field in the infinite matrix containing only one of the cracks among the network presented in Fig. 8 (crack number 1). The pressure field, before this crack is placed in the matrix, is represented by the straight line corresponding to the farfield gradient. The bold line displays the pressure field on line containing a first crack placed in the matrix and the two other lines, the pressure on the lines parallel to the first line and distant from it of $h$ and $2 h$. When the second and third cracks are placed in the matrix at a distance $h$ and $2 h$ from the first crack (cracks number 2 and 3 in Fig. 8), they are placed in the pressure field locally represented by the curve $y=h$ and $y=2 h$ in the figure, hence, in a local pressure field with smaller gradients compared to the farfield gradient encountered by the first crack. The $\int_{\Gamma} q d s$ determining the contribution of these cracks to the global permeability is therefore smaller than that obtained for the first crack. This reasoning is somehow approximate since it takes into account the effect of the presence of the first crack on the pressure field encountered by the following cracks but not the effects of the following cracks on the first one. However, it explains well why the crack interaction tends to decrease the slope of the equivalent permeability curve versus the crack density, as found by the numerical results shown in Fig. 7. It also confirms that the self consistent scheme is here deficient. 


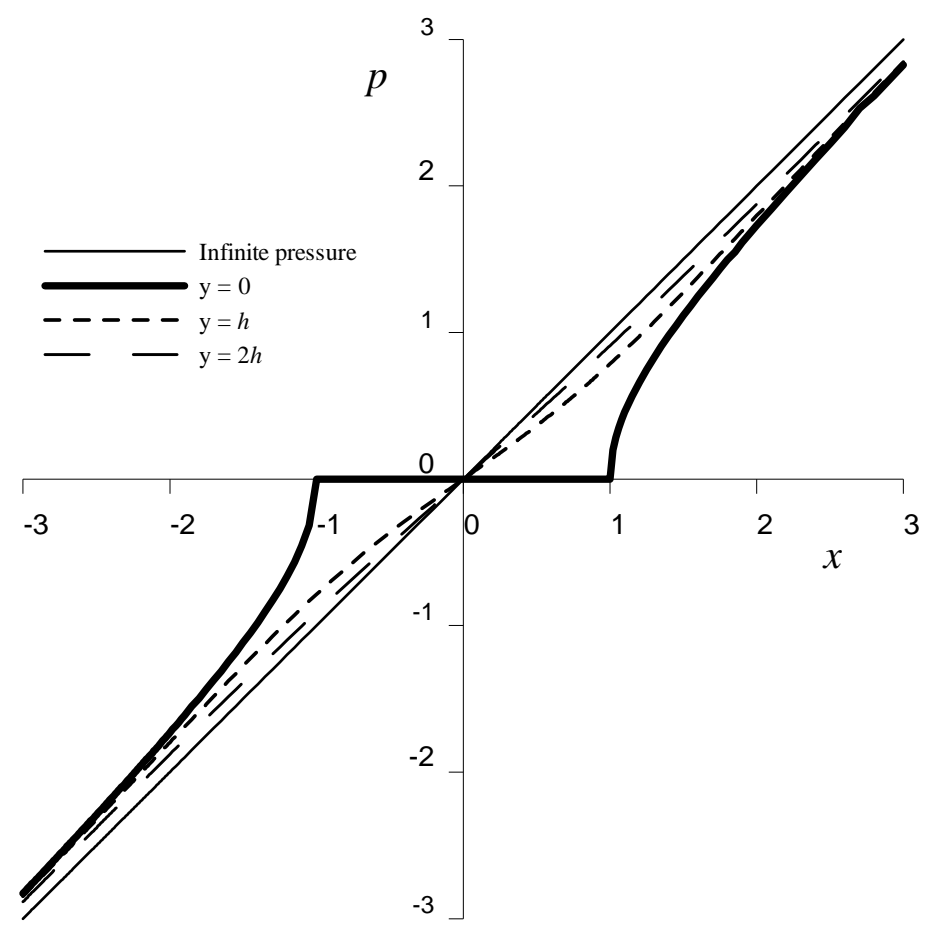

Fig. 10 Pressures along the lines at different level of $y$ these are parallel to the axe $x$ on which an isolated crack is placed $(\lambda=\infty)$

\section{Conclusions}

Recent theoretical advances in the modelling of flow in cracked porous materials (Pouya and Ghabezloo, 2010) were in some points completed here and then used to establish a numerical method for the study of these materials. This method was validated in the simple cases of a finite size crack in an infinite matrix for which closed-form solutions are available. It was shown that this method can be easily applied to the determination of the steady state flow in the materials with an anisotropic permeability, containing a dense distribution of curved-line cracks that intersect together. Some examples showed the efficiency of this method to determine the equivalent permeability of micro-cracked porous materials.

The efficiency of the numerical method is partly due to special choice of collocation points and to using closed-form expressions of elementary integrals. The theoretical result obtained on the mass balance at intersection points allows establishing a probably more efficient numerical method in which nodes are chosen as collocation points and using yet analytical expressions for elementary integrals. Furthermore, the theoretical results and the numerical algorithm may be extended to the 3D steady-state flow problem. These extensions are pursued in our ongoing research and will be presented in future papers.

Although these methods include some simplificative assumptions, like the uniform permeability of the matrix and no pressure jump between the two faces of the fracture, they can be usefully applied to many current research and engineering problems. The determination of the equivalent permeability of fractured oil reservoirs, of geological 
formations used for $\mathrm{CO}_{2}$ sequestration and of damaged zones around underground galleries for nuclear waste disposal are some possible examples of applications that we undertake in parallel researches.

\section{Appendix: Analytical expression of elementary integrals}

The element $E_{n}$ between the endpoints $\underline{z}_{1}, \underline{z}_{2}$ is considered with the unit tangent vector designated by $\underline{t}$. An arbitrary point in the plane of the crack is designated by $\underline{x}$. It can be shown that the elementary integral in Eq.(13) can be calculated analytically with the interpolation functions (15)-(17) for current elements and extremity elements as

following:

Current element

$$
I_{n}(\underline{x})=\frac{c}{2} \log \frac{\left(\underline{x}-\underline{z}_{2}\right) \cdot \mathbf{k}^{-1} \cdot\left(\underline{x}-\underline{z}_{2}\right)}{\left(\underline{x}-\underline{z}_{1}\right) \cdot \mathbf{k}^{-1} \cdot\left(\underline{x}-\underline{z}_{1}\right)} \frac{p_{2}-p_{1}}{L}
$$

Extremity element

$$
I_{n}(\underline{x})=\varepsilon \frac{3 c}{2}\left(2+\alpha \log \Phi+\beta \operatorname{Arctan} \frac{2 \beta L}{L-\zeta}\right) \frac{p_{2}-p_{1}}{L}
$$

with

$$
\tau=\underline{t} \cdot \mathbf{k}^{-1} \cdot \underline{t}, \zeta=\sqrt{\frac{\left(\underline{x}-\underline{z}_{0}\right) \cdot \mathbf{k}^{-1} \cdot\left(\underline{x}-\underline{z}_{0}\right)}{\underline{t} \cdot \mathbf{k}^{-1} \cdot \underline{t}}}, \phi=\varepsilon \frac{\left(\underline{x}-\underline{z}_{0}\right) \cdot \mathbf{k}^{-1} \cdot \underline{t}}{\underline{t} \cdot \mathbf{k}^{-1} \cdot \underline{t}}
$$

and

$$
\alpha=\sqrt{\frac{\zeta+\phi}{2 L}}, \beta=\sqrt{\frac{\zeta-\phi}{2 L}} \text { and } \Phi=\frac{\sqrt{L^{2}-2 \phi L+\zeta^{2}}}{L+\sqrt{2(\zeta+\phi) L}+\zeta}
$$

where $c$ stands for the hydraulic conductivity of crack; $p_{1}, p_{2}$ the nodal pressures of the element $E_{n}, \underline{z}_{0}=\underline{z}_{1}$ and $\varepsilon=1$ for the left extremity element, $\underline{z}_{0}=\underline{z}_{2}$ and $\varepsilon=-1$ for the right extremity elements.

\section{References}

Barthélémy, J.-F.: Effective permeability of media with a dense network of long and micro fractures. Transp Porous Media 76, 153-178 (2009)

Billaux, D.: Hydrogéologie des milieux fracturés. Géométrie, connectivité et comportement hydraulique. Thèse de doctorat, Ecole Nationale Supérieure des Mines de Paris (1990)

Bogdanov, I.I., Mourzenko, V.V., Thovert, J.F., Adler, P.M.: Effective permeability of fractured porous media in steady state flow. Water Resources Research 39(1),1-16 (2003)

Bogdanov, I.I., Mourzenko, V.V., Thovert, J.F., Adler, P.M.: Effective permeability of fractured porous 
media with power-law distribution of fracture sizes. Phys. Rev. E 76, 036309 (2007)

Bonnet, M.: Equations Intégrales et Éléments de Frontière. CNRS Editions/Eyrolles, Paris (1995)

Dormieux, L., Kondo, D.: Approche micromécanique du couplage perméabilité-endommagement. C.R.Mecanique 332, 135-140 (2004)

Gervais, F.,: Modélisation géométrique d'un réseau de fractures dans un massif rocheux stratifié. Application aux carrières marbrières de Comblanchien (Côte d'Or, France). PhD dissertation. Ecole Nationale Supérieure des Mines de Paris (1993)

Goméz-Hernández, J.J., Wen, X.H.: Upscaling hydraulic conductivities in heterogeneous media: an overview. J. Hydrol. 183, ix-xxxii (1996)

Guéguen, Y., Palciauskas, V.: Introduction to Physics of Rocks. Princeton University Press, Princeton (1994)

Guiggiani, M.: The Evaluation of Cauchy Principal Value Integrals in the Boundary Element Method - A Review. Mathematical and Computer Modelling. 15 (3-5), 175-184 (1990)

Guiggiani, M., Krishnasamy, G., Rudolphi T.J., Rizzo F.J.: A general algorithm for the numerical solution of hypersingular boundary integral equations. ASME J. of Applied Mechanics. 59, 604-614 (1990)

Koudina, N., R. Gonzalez Garcia, R., Thovert, J.F., Adler, P.M.: Permeability of three-dimensional fracture networks. Phys. Rev. E 57, 4466-4479 (1998)

Liolios, P.A., Exadaktylos, G.E.: A solution of steady-state fluid flow in multiply fractured isotropic porous media. Int. J. Solids Struct. 43, 3960-3982 (2006)

Long J.C.S., Remer J.S.: Wilson C.R.: Witherspoon P.A: Porous media equivalents for networks of discontinuous fractures, Water Resources Research 18 (3), 645-658 (1982)

Louis, C.: A study of groundwater flow in jointed rock and its influence on the stability of rock mass, Imperial College Rock Mechanic. Report, 10 (1969)

Manzocchi T., Childs C., Walsh J.J.: Faults and fault properties in hydrocarbon flow models. Geofluids (2010), 10, 94-113.

Muskhelishvili, N.I.: Singular Integral Equations. P. Noordhoff Ltd., Groningen, Holland (1953)

Mourzenko, V.V., Thovert, J.F., Adler, P.M.: Permeability of isotropic and anisotropic fracture networks, from the percolation threshold to very large densities. Phys. Rev. E 84, 036307 (2011)

Norton, D. L., Knapp, R.: Transport phenomena in hydrothermal systems: the nature of porosity, American J. of Science, 277, 913-36 (1977)

Pouya, A., Fouché, O.: Permeability of 3D discontinuity networks: new tensors from boundaryconditioned homogenization. Adv. Water Resour. 32, 303-314 (2009)

Pouya, A., Ghabezloo, S.: Flow around a crack in a porous matrix and related problems. Transport in Porous Media 84(2), 511-532 (2010)

Renard, P., de Marsily, G.: Calculating equivalent permeability: a review. Adv.Water Resour. 20(5-6), 253-278 (1997)

Sánchez-Vila, X., Girardi, G.P., Carrera, J.: A synthesis of approaches to upscaling of hydraulic conductivities. Water Resour. Res. 31(4), 867-882 (1995)

Shafiro, B., Kachanov, .M: Anisotropic effective conductivity of material with nonrandomly oriented inclusions of diverse ellipsoidal shapes. Journal of applied physics. 87(12), 8561-8569 (2000)

Sutradhar, A., Paulino, G. H., Gray, L. J.: Symmetric Galerkin Boundary Element Method. Springer (2008)

Tanaka, M., Saldek, V., Saldek, J.: Regularization techniques applied to boundary element methods. Appl Mech Rev. 47(10), 457-499 (1994)

Witherspoon, P. A., Wang, J. S. Y., Iwai, K., Gale, J. E.: Validity of cubic law for fluid flow in a deformable rock fracture. Water Resour. Res. 16, 1016-24 (1980)

Zimmerman, R. W., Effective conductivity of a two-dimensional medium containing elliptical inhomogeneities. Proc. R. Soc. London, Ser. A, 452, 1713- 1727 (1996) 